\title{
Double type II seesaw mechanism accompanied by Dirac fermionic dark matter
}

\author{
Pei-Hong $\mathrm{Gu}^{1,2, *}$ \\ ${ }^{1}$ Department of Physics, Southeast University, Nanjing 211189, China \\ ${ }^{2}$ School of Physics and Astronomy, Shanghai Jiao Tong University, \\ 800 Dongchuan Road, Shanghai 200240, China
}

(Received 1 August 2019; revised manuscript received 5 November 2019; published 9 January 2020)

In the type II seesaw mechanism, the neutrino mass generation could be tested experimentally if the Higgs triplet is at the TeV scale and has a small cubic coupling to the standard model Higgs doublet. We show such small triplet-doublet coupling and the cosmic baryon asymmetry can be simultaneously induced by an additional seesaw mechanism involving a $U(1)_{B-L}$ gauge symmetry. Meanwhile, three right-handed neutrinos for canceling the gauge anomalies can form a stable Dirac fermionic dark matter besides an acceptably massless fermion.

DOI: 10.1103/PhysRevD.101.015006

\section{INTRODUCTION}

The atmospheric, solar, accelerator, and reactor neutrino experiments have established the phenomenon of neutrino oscillations. Three flavors of neutrinos thus should be massive and mixed [1]. Moreover, the neutrinos should be extremely light to fulfill the cosmological observations [1]. The tiny but nonzero neutrino masses call for new physics beyond the standard model (SM). Currently, the best explanation for the small neutrino masses seems to be the famous seesaw [2] mechanism. In some seesaw models $[2,3]$, the interactions for generating the small neutrino masses can also produce a lepton asymmetry stored in the SM leptons [4-13]. The sphaleron [14] processes then can partially convert the produced lepton asymmetry to a baryon asymmetry. This means the cosmic baryon asymmetry, which is another big challenge to the SM, can have a common origin with the small neutrino masses.

The type II seesaw [3] has become one of the most attractive seesaw scenarios. In the type II seesaw models, the Higgs triplet has a cubic coupling to the SM Higgs doublet. In the presence of a small triplet-doublet cubic coupling, the Higgs triplet can pick up a tiny vacuum expectation value (VEV) even if it is at the TeV scale. Accordingly, the Yukawa couplings of this $\mathrm{TeV}$-scale Higgs triplet to the SM lepton doublets can be allowed at a testable level $[15,16]$. Obviously, the key of the testable type II seesaw is the small cubic

\footnotetext{
*peihong.gu@sjtu.edu.cn
}

Published by the American Physical Society under the terms of the Creative Commons Attribution 4.0 International license. Further distribution of this work must maintain attribution to the author(s) and the published article's title, journal citation, and DOI. Funded by SCOAP ${ }^{3}$. coupling between the Higgs triplet and doublet. In an early work [17], we introduced a global symmetry of lepton number to simultaneously explain such small triplet-doublet coupling and the cosmic baryon asymmetry. However, the spontaneous breaking scale of the global lepton number is quite arbitrary.

In this paper we shall realize a double type II seesaw mechanism by resorting to a $U(1)_{B-L}$ gauge symmetry which forbids the Yukawa couplings of three right-handed neutrinos to the SM. Through their Yukawa couplings to a Higgs singlet for spontaneously breaking the $U(1)_{B-L}$ symmetry, the right-handed neutrinos eventually can form a Dirac fermion to become a stable dark matter particle besides a harmlessly massless state. Because of this $U(1)_{B-L}$ symmetry breaking, two or more heavy Higgs singlets can acquire their small VEVs to suppress the cubic coupling between the usual type II seesaw Higgs triplet and the SM Higgs doublet. Therefore, the left-handed neutrinos can naturally obtain their tiny Majorana masses even if the Higgs triplet with sizable Yukawa couplings is set at the $\mathrm{TeV}$ scale. Our model can also accommodate a successful leptogenesis mechanism through the heavy Higgs singlet decays.

\section{FERMIONS AND SCALARS}

The SM fermions and scalar are denoted as follows,

$$
\begin{array}{ll}
q_{L}\left(3,2,+\frac{1}{6},+\frac{1}{3}\right), & d_{R}\left(3,1,-\frac{1}{3},+\frac{1}{3}\right), \\
u_{R}\left(3,1,+\frac{2}{3},+\frac{1}{3}\right) ; & l_{L}\left(1,2,-\frac{1}{2},-1\right), \\
e_{R}(1,1,-1,-1) ; & \phi\left(1,2,-\frac{1}{2}, 0\right) .
\end{array}
$$


Here and thereafter the brackets following the fields describe the transformations under the $S U(3)_{c} \times S U(2)_{L} \times$ $U(1)_{Y} \times U(1)_{B-L}$ gauge groups. For simplicity, we do not show the indices of the three generations of fermions. In order to cancel the gauge anomalies, we need some righthanded neutrinos with appropriate $U(1)_{B-L}$ charges $[18,19]$. In the present work, we shall consider the following three right-handed neutrinos [18],

$\nu_{R 1}(1,1,0,-4), \quad \nu_{R 2}(1,1,0,-4), \quad \nu_{R 3}(1,1,0,+5)$.

The Higgs doublet $\phi$ is responsible for the electroweak symmetry breaking as usual. We then introduce a Higgs singlet,

$$
\xi(1,1,0,+1),
$$

for spontaneously breaking the $U(1)_{B-L}$ symmetry. Moreover, the model contains other Higgs scalars including a Higgs triplet,

$$
\Delta(1,3,+1,+2),
$$

and two or more heavy Higgs singlets,

$$
\sigma_{a}(1,1,0,-2), \quad(a=1, \ldots, n \geq 2) .
$$

For simplicity, we do not write down the full Lagrangian. Instead, we only give the following terms,

$$
\begin{aligned}
\mathcal{L} \supset & -\mu_{\xi}^{2} \xi^{\dagger} \xi-\lambda_{\xi}\left(\xi^{\dagger} \xi\right)^{2}-\lambda_{\xi \phi} \xi^{\dagger} \xi \phi^{\dagger} \phi \\
& -\left(\mu_{\Delta}^{2}+\lambda_{\xi \Delta} \xi^{\dagger} \xi+\lambda_{\phi \Delta} \phi^{\dagger} \phi\right) \operatorname{Tr}\left(\Delta^{\dagger} \Delta\right) \\
& -\sum_{a=1}^{n}\left(M_{a}^{2} \sigma_{a}^{\dagger} \sigma_{a}+\kappa_{a} \sigma_{a} \phi^{T} i \tau_{2} \Delta \phi+\mu_{a} \sigma_{a} \xi^{2}\right) \\
& -\frac{1}{2} f \bar{l}_{L}^{c} i \tau_{2} \Delta l_{L}-\sum_{i=1,2} y_{i 3} \xi \bar{\nu}_{R i} \nu_{R 3}^{c}+\text { H.c. }
\end{aligned}
$$

Note one of the four couplings $\kappa_{a, b \neq a}$ and $\mu_{a, b \neq b}$ can always keep complex after taking any phase rotations.

We could replace the heavy Higgs singlets $\sigma_{a}$ in the above model by some heavy Higgs triplets,

$\Omega_{a}(1,3,+1,+1)$ or $\Psi_{a}(1,3,+1,0), \quad(a=1, \ldots, n \geq 2)$.

The third line in Eq. (6) then should be modified by

$$
\begin{aligned}
\mathcal{L} \supset & -\sum_{a=1}^{n}\left[M_{a}^{2} \operatorname{Tr}\left(\Omega_{a}^{\dagger} \Omega_{a}\right)+\kappa_{a} \xi^{\dagger} \phi^{T} i \tau_{2} \Omega_{a} \phi+\mu_{a} \xi \operatorname{Tr}\left(\Delta^{\dagger} \Omega_{a}\right)\right] \\
& + \text { H.c. } \\
\mathcal{L} \supset & -\sum_{a=1}^{n}\left[M_{a}^{2} \operatorname{Tr}\left(\Psi_{a}^{\dagger} \Psi_{a}\right)+\mu_{a} \phi^{T} i \tau_{2} \Psi_{a} \phi+\kappa_{a} \xi^{2} \operatorname{Tr}\left(\Delta^{\dagger} \Psi_{a}\right)\right] \\
& + \text { H.c. }
\end{aligned}
$$

Actually, the terms (8) was mentioned in our early work [17], where a global symmetry of lepton number was introduced rather than the $U(1)_{B-L}$ gauge symmetry. In a later work [20], the authors studied the terms (9) with only one heavy Higgs triplet $\Psi_{1}$. In this simple case, it seemed difficult to well motivate the small mixing [21] between the heavy Higgs triplet $\Psi_{1}$ and the TeV-scale Higgs triplet $\Delta$ since a successful leptogenesis required at least two heavy Higgs triplets $\Psi_{a}$. Moreover, the right-handed neutrinos for canceling the gauge anomaly did not appear in their demonstration.

In the following we shall focus on the model (6). The alternative models (8) and (9) will be studied elsewhere.

\section{NEUTRINO MASS}

The $U(1)_{B-L}$ symmetry breaking is expected before the electroweak symmetry breaking. The Higgs singlet $\xi$ and the Higgs doublet $\phi$ are responsible for spontaneously breaking the $U(1)_{B-L}$ symmetry and the electroweak symmetry respectively. When the $U(1)_{B-L}$ symmetry is spontaneously broken while the electroweak symmetry is still conserved, we can minimize the scalar potential to be

$$
\begin{aligned}
\frac{\partial V}{\partial\langle\xi\rangle} & =\langle\xi\rangle\left(2 \mu_{\xi}^{2}+4 \lambda_{\xi}\langle\xi\rangle^{2}+2 \mu_{a}\left\langle\sigma_{a}\right\rangle+2 \mu_{a}^{*}\left\langle\sigma_{a}^{*}\right\rangle\right) \\
& =0, \\
\frac{\partial V}{\partial\left\langle\sigma_{a}^{*}\right\rangle} & =M_{a}^{2}\left\langle\sigma_{a}\right\rangle+\mu_{a}^{*}\langle\xi\rangle^{2}=0 .
\end{aligned}
$$

In the limiting case of $\left\langle\sigma_{a}\right\rangle,\left\langle\sigma_{a}^{*}\right\rangle \ll\langle\xi\rangle$, we can obtain

$$
\langle\xi\rangle \simeq \sqrt{-\frac{\mu_{\xi}^{2}}{2 \lambda_{\xi}}} \text { for } \mu_{\xi}^{2}<0, \quad \lambda_{\xi}>0,
$$

as well as

$$
\left\langle\sigma_{a}\right\rangle \simeq-\frac{\mu_{a}^{*}\langle\xi\rangle^{2}}{M_{a}^{2}} \ll\langle\xi\rangle \text { for } M_{a} \gtrsim \mu_{a}, \quad M_{a} \gg\langle\xi\rangle .
$$

We hence can obtain a cubic coupling between the Higgs triplet $\Delta$ and the SM Higgs doublet $\phi$, i.e.,

$\mathcal{L} \supset-\rho \phi^{T} i \tau_{2} \Delta \phi+$ H.c. $\quad$ with $\quad \rho=\sum_{a} \rho_{a}=\sum_{a} \kappa_{a}\left\langle\sigma_{a}\right\rangle$.

From this triplet-doublet coupling, the Higgs triplet $\Delta$ can pick up a small VEV $\langle\Delta\rangle$ after the Higgs doublet $\phi$ acquires its VEV $\langle\phi\rangle \simeq 174 \mathrm{GeV}$ for the electroweak symmetry breaking, 


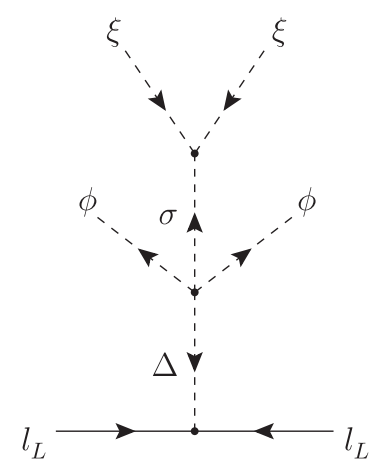

FIG. 1. The neutrino mass generation.

$\langle\Delta\rangle \simeq-\frac{\rho^{*}\langle\phi\rangle^{2}}{M_{\Delta}^{2}} \ll\langle\phi\rangle$ for $M_{\Delta} \gg \rho, \quad M_{a} \gtrsim\langle\phi\rangle$.

Here the Higgs triplet mass $M_{\Delta}$ is dominated by

$$
M_{\Delta}^{2} \simeq \mu_{\Delta}^{2}+\lambda_{\xi \Delta}\langle\xi\rangle^{2}+\lambda_{\phi \Delta}\langle\phi\rangle^{2} .
$$

In the presence of the Higgs triplet $\operatorname{VEV}\langle\Delta\rangle$, the lefthanded neutrinos $\nu_{L}$ can obtain their Majorana masses,

$$
\mathcal{L} \supset-\frac{1}{2} m_{\nu} \bar{\nu}_{L}^{c} \nu_{L}+\text { H.c. } \quad \text { with } \quad m_{\nu}=f\langle\Delta\rangle .
$$

If the $\operatorname{VEV}\langle\Delta\rangle$ is at the $\mathrm{eV}$ scale, we can take the Yukawa couplings $f$ to be sizable and then yield the Majorana neutrino masses $m_{\nu}$ at a desired level. The diagram for the neutrino mass generation is shown in Fig. 1.

For a numerical example, we first fix $\langle\xi\rangle=21 \mathrm{TeV}$ and then input $M_{a}=10^{14} \mathrm{GeV}, \mu_{a}=2 \times 10^{12} \mathrm{GeV}, \kappa_{a}=0.2$. Consequently, we can have $\rho_{a}=18 \mathrm{eV}$. By further inputting $M_{\Delta}=\mathcal{O}(\mathrm{TeV})$, we can obtain $\langle\Delta\rangle=\mathcal{O}(0.1-1 \mathrm{eV})$ and hence $m_{\nu}=\mathcal{O}(0.01-0.1 \mathrm{eV})$ for $f=\mathcal{O}(0.01-1)$.

The generation of the small Higgs triplet $\operatorname{VEV}\langle\Delta\rangle$ and hence the tiny neutrino masses $m_{\nu}$ is the so-called type II seesaw. For inducing the small VEV $\langle\Delta\rangle$, the Higgs triplet
$\Delta$ should have a suppressed cubic coupling $\rho$ with the SM Higgs doublet $\phi$ if the Higgs triplet $\Delta$ has a mass $M_{\Delta}$ at $\mathrm{TeV}$ scale. In the present work, the triplet-doublet coupling $\rho$ can be naturally achieved in an additional seesaw way, where the heavy Higgs singlets $\sigma_{a}$ are far above the $U(1)_{B-L}$ gauge symmetry breaking scale $\langle\xi\rangle$. Therefore, we may name this two-step seesaw mechanism as a double type II seesaw [17].

When the TeV-scale Higgs triplet $\Delta$ for the type II seesaw has a tiny VEV, it can have the sizable Yukawa couplings to the SM lepton doublets $l_{L}$. Such Yukawa couplings then could be sensitive to some lepton flavor violating processes such as $\mu \rightarrow e e e$ and $\mu \rightarrow e \gamma$. The comprehensive analysis can be found in [15]. The TeVscale type II seesaw could be also systematically tested at the LHC [16].

\section{BARYON ASYMMETRY}

As shown in Fig. 2, the heavy Higgs singlets $\sigma_{a}$ have two decay modes,

$$
\sigma_{a} \rightarrow \phi^{*} \phi^{*} \Delta^{*}, \quad \sigma_{a} \rightarrow \xi^{*} \xi^{*} .
$$

As long as the $C P$ is not conserved, we can expect a $C P$ asymmetry in the above decays,

$$
\begin{aligned}
\varepsilon_{a} & =2 \frac{\Gamma\left(\sigma_{a} \rightarrow \phi^{*} \phi^{*} \Delta^{*}\right)-\Gamma\left(\sigma_{a}^{*} \rightarrow \phi \phi \Delta\right)}{\Gamma_{a}} \\
& =-2 \frac{\Gamma\left(\sigma_{a} \rightarrow \xi^{*} \xi^{*}\right)-\Gamma\left(\sigma_{a}^{*} \rightarrow \xi \xi\right)}{\Gamma_{a}} \neq 0,
\end{aligned}
$$

where $\Gamma_{a}$ is the total decay width,

$$
\begin{aligned}
\Gamma_{a} & =\Gamma\left(\sigma_{a} \rightarrow \phi^{*} \phi^{*} \Delta^{*}\right)+\Gamma\left(\sigma_{a} \rightarrow \xi^{*} \xi^{*}\right) \\
& =\Gamma\left(\sigma_{a}^{*} \rightarrow \phi \phi \Delta\right)+\Gamma\left(\sigma_{a}^{*} \rightarrow \xi \xi\right) .
\end{aligned}
$$

We can calculate the decay width at tree level and the $C P$ asymmetry at one-loop level,

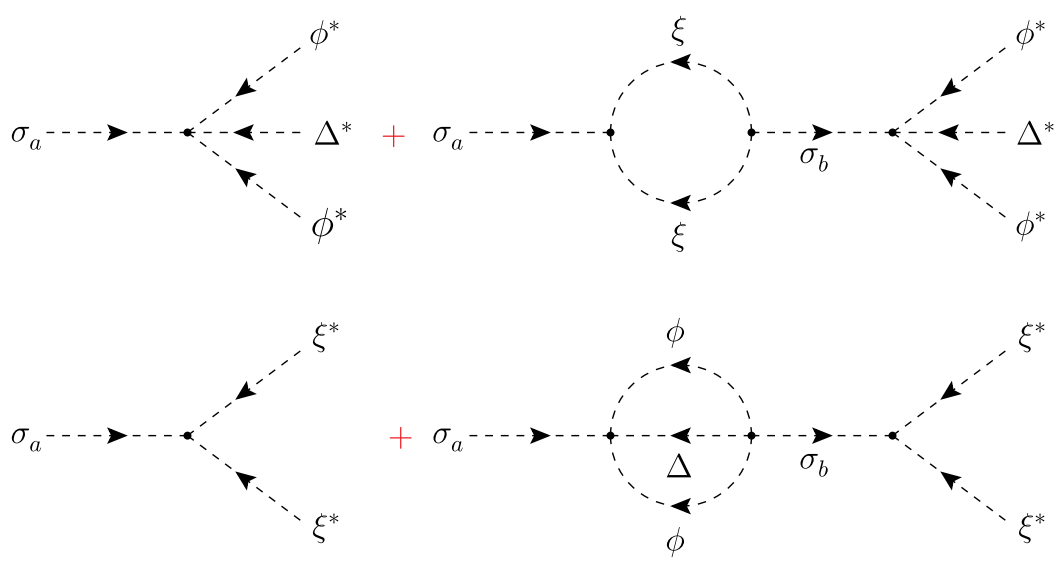

FIG. 2. The heavy Higgs singlet decays. 


$$
\begin{aligned}
\Gamma_{a} & =\frac{1}{8 \pi}\left(\frac{\left|\mu_{a}\right|^{2}}{M_{a}^{2}}+\frac{3}{32 \pi^{2}}\left|\kappa_{a}\right|^{2}\right) M_{a}, \\
\varepsilon_{a} & =-\frac{3}{32 \pi^{3}} \sum_{b \neq a} \frac{\operatorname{Im}\left(\kappa_{a}^{*} \kappa_{b} \mu_{a} \mu_{b}^{*}\right)}{\frac{\left|\mu_{a}\right|^{2}}{M_{a}^{2}}+\frac{3\left|\kappa_{a}\right|^{2}}{32 \pi^{2}}} \frac{1}{M_{b}^{2}-M_{a}^{2}} \\
& =-\frac{3}{32 \pi^{3}} \sum_{b \neq a} \frac{\left|\kappa_{a} \kappa_{b} \mu_{a} \mu_{b}\right| \sin \delta_{a b}}{\frac{\left|\mu_{a}\right|^{2}}{M_{a}^{2}}+\frac{3\left|\kappa_{a}\right|^{2}}{32 \pi^{2}}} \frac{1}{M_{b}^{2}-M_{a}^{2}} .
\end{aligned}
$$

Here $\delta_{a b}$ is the relative phase among the parameters $\rho_{a, b}$ and $\kappa_{a, b}$.

After the heavy Higgs singlets $\sigma_{a}$ go out of equilibrium, their decays can generate a lepton asymmetry $L_{\Delta}$ stored in the Higgs triplet $\Delta$. For demonstration, we simply assume the heavy Higgs singlet $\sigma_{1}$ much lighter than the other heavy Higgs singlets $\sigma_{2, \ldots}$. In this case, the $\sigma_{1}$ decays should dominate the final $L_{\Delta}$ asymmetry, i.e.,

$$
L_{\Delta}=\frac{\varepsilon_{1}}{g_{*}} \kappa,
$$

with $\kappa<1$ being a washout factor and $g_{*}=122$ being the relativistic degrees of freedom (the SM fields plus the three right-handed neutrinos $\nu_{R}$, the Higgs triplet $\Delta$, the Higgs singlet $\xi$ and the $U(1)_{B-L}$ gauge field.). To determine the $\kappa$ factor, we need give and solve the Boltzmann equations. We shall do with the Boltzmann equations in a future work including the present model (6) as well as the alternative models (8) and (9). In the present work, we shall simply consider the weak washout case where the decay width $\Gamma_{1}$ is required to fulfill the condition as below,

$$
K=\left.\frac{\Gamma_{1}}{H(T)}\right|_{T=M_{1}} \ll 1 .
$$

Here and thereafter $H(T)$ is the Hubble constant,

$$
H(T)=\left(\frac{8 \pi^{3} g_{*}}{90}\right)^{\frac{1}{2}} \frac{T^{2}}{M_{\mathrm{Pl}}},
$$

with $M_{\mathrm{Pl}} \simeq 1.22 \times 10^{19} \mathrm{GeV}$ being the Planck mass. In this weak washout case, the heavy Higgs singlet $\sigma_{1}$ can begin their out-of-equilibrium decays when they become nonrelativistic, i.e., $T \sim M_{1}$. The $L_{\Delta}$ asymmetry then can be well described by

$$
\begin{aligned}
& \left.L_{\Delta} \simeq \varepsilon_{1}\left(\frac{n_{\sigma_{1}}^{e q}}{s}\right)\right|_{T=M_{1}} \text { with } \\
& n_{\sigma_{1}}^{e q}=2\left(\frac{M_{1} T}{2 \pi}\right)^{\frac{3}{2}} e^{-\frac{M_{1}}{T}, \quad s=\frac{2 \pi^{2}}{45} g_{*} T^{3},}
\end{aligned}
$$

where the symbol $n_{\sigma_{1}}^{e q}$ is the equilibrium number density of the heavy Higgs singlets $\sigma_{1}$, while the character $s$ is the entropy density of the universe [22]. Comparing with Eq. (23), the washout factor $\kappa$ is given by

$$
\kappa \simeq \frac{45}{2^{\frac{3}{2}} \pi^{\frac{7}{2}} e}=0.11
$$

In the above estimations, the related $U(1)_{B-L}$ gauge interactions have been assumed to decouple before the decays. For this purpose, we can take the heavy Higgs singlet $\sigma_{1}$ heavy enough and hence the processes involving the gauge interactions can have a rate $\Gamma_{g}$ smaller than the decay width $\Gamma_{1}$, i.e.,

$$
\Gamma_{g} \sim \frac{g_{B-L}^{4}}{M_{1}^{2}} n_{\sigma_{1}}^{e q}<\Gamma_{1}
$$

with $g_{B-L}$ being the $U(1)_{B-L}$ gauge coupling.

The cubic coupling between the Higgs triplet and doublet can go into equilibrium at a temperature much below the electroweak scale even if it appears before the electroweak symmetry breaking. Actually, we can estimate the interaction rate of the triplet-doublet coupling,

$$
\Gamma_{\phi \phi \Delta} \sim \begin{cases}\frac{|\rho|^{2}}{T} & \text { for } T>M_{\Delta}, \\ \frac{|\rho|^{2}}{M_{\Delta}} & \text { for } T<M_{\Delta},\end{cases}
$$

and then require it to keep smaller than the Hubble constant $H(T)$ above the electroweak scale, i.e.,

$$
\Gamma_{\phi \phi \Delta}<H(T) \text { for } T \gtrsim 100 \mathrm{GeV} .
$$

The above condition can be achieved for $|\rho| \lesssim \mathcal{O}(\mathrm{keV})$ and $M_{\Delta}=\mathcal{O}(\mathrm{TeV})$. Therefore, the lepton asymmetry $L_{\Delta}$ stored in the Higgs triplet $\Delta$ will become a lepton asymmetry stored in the SM lepton doublets $l_{L}$ before the electroweak symmetry breaking, thanks to the fast decays of the Higgs triplet into the lepton doublets. Below the crucial temperature $T_{\mathrm{sph}} \sim 10^{12} \mathrm{GeV}$, the sphaleron processes then can partially transfer this lepton asymmetry to a baryon asymmetry [23],

$$
B=-\frac{28}{79} L_{\Delta}
$$

Here we have simply assumed the lepton asymmetry $L_{\Delta}$ to be induced at the very high temperatures $T>T_{\mathrm{sph}} \sim$ $10^{12} \mathrm{GeV}$. Accordingly, the Yukawa interactions between the SM left- and right-handed leptons have not been in equilibrium so that we could safely ignore the so-called flavor effect [8].

As a numerical example, we take $M_{1}=10^{14} \mathrm{GeV}$, $\left|\mu_{1}\right|=2 \times 10^{12} \mathrm{GeV},\left|\kappa_{1}\right|=0.2 ; M_{2}=10^{15} \mathrm{GeV},\left|\mu_{2}\right|=$ $2 \times 10^{13} \mathrm{GeV},\left|\kappa_{2}\right|=0.2$. The $C P$ asymmetry $\varepsilon_{1}$ then should be $\varepsilon_{1}=-6.2 \times 10^{-6} \sin \delta_{12}$. Meanwhile, the weak washout condition can be satisfied [22], i.e., $K=0.2$. 
The baryon number hence can be approximately given by [22]

$B=-\frac{28}{79} L_{\Delta} \simeq-\frac{28}{79} \frac{\varepsilon_{1}}{g_{*}} \times 0.11=10^{-10}\left(\frac{\sin \delta_{12}}{0.05}\right)$.

\section{DARK FERMIONS}

As shown in Eq. (6), the three right-handed neutrinos $\nu_{R}$ have two Yukawa couplings with the $U(1)_{B-L}$ Higgs singlet $\xi$, i.e.,

$$
\begin{aligned}
\mathcal{L} & \supset-\left[\begin{array}{ll}
\bar{\nu}_{R 1} & \bar{\nu}_{R 2}
\end{array}\right]\left[\begin{array}{l}
y_{13} \\
y_{23}
\end{array}\right] \nu_{R 3}^{c} \xi+\text { H.c. } \\
& =-\left[\begin{array}{ll}
\bar{\zeta}_{R} & \bar{\chi}_{R}
\end{array}\right]\left[\begin{array}{c}
0 \\
y_{\chi}
\end{array}\right] \nu_{R 3}^{c} \xi+\text { H.c. with } \\
\zeta_{R} & =\frac{y_{23} \nu_{R 1}-y_{13} \nu_{R 2}}{\sqrt{y_{13}^{2}+y_{23}^{2}}}, \quad \chi_{R}=\frac{y_{13} \nu_{R 1}+y_{23} \nu_{R 2}}{\sqrt{y_{13}^{2}+y_{23}^{2}}}, \\
y_{\chi} & =\sqrt{y_{13}^{2}+y_{23}^{2}} .
\end{aligned}
$$

So, the third right-handed neutrino $\nu_{R 3}$ and the linear combination $\chi_{R}$ of the two right-handed neutrinos $\nu_{R 1,2}$ can form a Dirac particle after the $U(1)_{B-L}$ symmetry breaking, i.e.,

$\mathcal{L} \supset i \bar{\chi} \gamma^{\mu} \partial_{\mu} \chi-m_{\chi} \bar{\chi} \chi$ with $\chi=\chi_{R}+\nu_{R 3}^{c}, \quad m_{\chi}=y_{\chi}\langle\xi\rangle$.

Meanwhile, the other linear combination $\zeta_{R}$ of the two right-handed neutrinos $\nu_{R 1,2}$ has no Yukawa couplings so that it should be a massless state. In consequence, the kinematics only allows the massive $\chi$ can annihilate (rather than decay) into the massless $\zeta_{R}$ through the mediation of the $U(1)_{B-L}$ gauge boson. This is like that in the basis of mass eigenstates, an SM fermion can only annihilate into the other SM fermions through the neutral current interaction or the Yukawa interaction. An SM fermion can decay into the other SM fermions only through the charged current interaction, which is not available for the present right-handed neutrinos $\nu_{R}$. Furthermore, the $U(1)_{B-L}$ gauge symmetry has protected the right-handed neutrinos $\nu_{R}$ from the other Yukawa couplings with the SM. So, the massive eigenstate $\chi$ of the right-handed neutrinos $\nu_{R}$ cannot decay into the SM particles. This means we can have a stable $\chi$.

Since the Dirac fermion $\chi$ is stable, it can be expected to serve as a dark matter particle. The dark matter annihilation and scattering could be determined by the gauge interactions,

$$
\begin{aligned}
\mathcal{L} & \supset g_{B-L} Z_{B-L}^{\mu}\left\{\sum_{i=1}^{3}\left(\frac{1}{3} \bar{d}_{i} \gamma_{\mu} d_{i}+\frac{1}{3} \bar{u}_{i} \gamma_{\mu} u_{i}-\bar{e}_{i} \gamma_{\mu} e_{i}-\bar{\nu}_{L i} \gamma_{\mu} \nu_{L i}\right)\right. \\
& -4 \bar{\zeta}_{R} \gamma_{\mu} \zeta_{R}-i 2 \operatorname{Tr}\left[\left(\partial_{\mu} \Delta\right)^{\dagger} \Delta-\text { H.c. }\right] \\
& \left.-\frac{1}{2} \bar{\chi} \gamma_{\mu}\left(9-\gamma_{5}\right) \chi\right\} .
\end{aligned}
$$

The gauge coupling $g_{B-L}$ then should have an upper bound from the perturbation requirement, i.e.,

$$
\frac{9}{2} g_{B-L}<\sqrt{4 \pi} \Rightarrow g_{B-L}<\frac{\sqrt{16 \pi}}{9},
$$

while the gauge boson mass $M_{Z_{B-L}}$ should be

$$
M_{Z_{B-L}}=\sqrt{2} g_{B-L}\langle\xi\rangle .
$$

The $Z_{B-L}$ contribution to the cross sections for $e^{+} e^{-} \rightarrow f \bar{f}$ proceeds through an $s$-channel $Z_{B-L}$ exchange (when $f=e$, there are also $t$ - and $u$-channels). In the case that $M_{Z_{B-L}}$ is above $209 \mathrm{GeV}$ (the maximum energy of LEP II), the bound on the $U(1)_{B-L}$ symmetry breaking scale should be [24-27],

$$
\frac{M_{Z_{B-L}}}{g_{B-L}} \gtrsim 7 \mathrm{TeV} \Rightarrow\langle\xi\rangle \gtrsim 5 \mathrm{TeV} .
$$

The thermally averaging dark matter annihilating cross section can be computed by [28]

$$
\begin{aligned}
\left\langle\sigma_{\mathrm{A}} v_{\text {rel }}\right\rangle= & \sum_{f=d_{i}, u_{i}, e_{i}, \nu_{L i}, \zeta_{R}}\left\langle\sigma\left(\chi+\chi^{c} \rightarrow f+f^{c}\right) v_{\text {rel }}\right\rangle \\
& +\left\langle\sigma\left(\chi+\chi^{c} \rightarrow \Delta+\Delta^{*}\right) v_{\text {rel }}\right\rangle \\
\simeq & \frac{2835 g_{B-L}^{4}}{8 \pi} \frac{m_{\chi}^{2}}{M_{Z_{B-L}}^{4}} \\
= & \frac{2835}{32 \pi} \frac{m_{\chi}^{2}}{\langle\xi\rangle^{4}}=\frac{2835}{32 \pi} \frac{y_{\chi}^{2}}{\langle\xi\rangle^{2}} .
\end{aligned}
$$

The dark matter relic density then can well approximate to

$$
\begin{aligned}
\Omega_{\chi} h^{2} & \simeq \frac{0.1 \mathrm{pb}}{\left\langle\sigma_{\mathrm{A}} v_{\text {rel }}\right\rangle}=0.1 \mathrm{pb} \times \frac{32 \pi\langle\xi\rangle^{4}}{2835 m_{\chi}^{2}} \\
& =0.1 \mathrm{pb} \times \frac{32 \pi\langle\xi\rangle^{2}}{2835 y_{\chi}^{2}} .
\end{aligned}
$$

It should be noted that Eqs. (39) and (40) are based on the assumption,

$4 m_{\chi}^{2} \ll M_{Z_{B-L}}^{2} \Rightarrow y_{\chi}^{2} \ll \frac{1}{2} g_{B-L}^{2}<\frac{8 \pi}{81} \Rightarrow y_{\chi}<\frac{\sqrt{8 \pi}}{9}$.

By inserting the upper bound (41) into Eq. (40), we can put a constraint on the $\operatorname{VEV}\langle\xi\rangle$, i.e., 


$$
\begin{aligned}
\langle\xi\rangle & \simeq\left(\frac{2835 y_{\chi}^{2} \Omega_{\chi} h^{2}}{32 \pi \times 0.1 \mathrm{pb}}\right)^{\frac{1}{2}} \\
& =61 \mathrm{TeV}\left(\frac{y_{\chi}}{\sqrt{8 \pi} / 9}\right)\left(\frac{\Omega_{\chi} h^{2}}{0.11}\right)^{\frac{1}{2}} \\
& <61 \mathrm{TeV}\left(\frac{\Omega_{\chi} h^{2}}{0.11}\right)^{\frac{1}{2}},
\end{aligned}
$$

besides the experimental limit (38). The dark matter mass,

$$
\begin{aligned}
m_{\chi} & \simeq\left(0.1 \mathrm{pb} \times \frac{32 \pi\langle\xi\rangle^{4}}{2835 \Omega_{\chi} h^{2}}\right)^{\frac{1}{2}} \\
& =4 \mathrm{TeV}\left(\frac{\langle\xi\rangle}{21 \mathrm{TeV}}\right)^{2}\left(\frac{0.11}{\Omega_{\chi} h^{2}}\right)^{\frac{1}{2}}
\end{aligned}
$$

thus should be in the range,

$$
\begin{aligned}
227 \mathrm{GeV}\left(\frac{0.11}{\Omega_{\chi} h^{2}}\right)^{\frac{1}{2}} \lesssim & m_{\chi}<34 \mathrm{TeV}\left(\frac{0.11}{\Omega_{\chi} h^{2}}\right)^{\frac{1}{2}} \\
& \text { for } 5 \mathrm{TeV} \lesssim\langle\xi\rangle<61 \mathrm{TeV} .
\end{aligned}
$$

The gauge interactions can also mediate the dark matter scattering off nucleons. The dominant spin-independent cross section is [29]

$$
\begin{aligned}
\sigma_{\chi N} & =\frac{81 g_{B-L}^{4}}{4 \pi} \frac{\mu_{r}^{2}}{M_{Z_{B-L}}^{4}} \\
& =\frac{81}{16 \pi} \frac{\mu_{r}^{2}}{\langle\xi\rangle^{4}}=\frac{2}{35} \frac{\mu_{r}^{2}}{m_{\chi}^{2}} \frac{0.1 \mathrm{pb}}{\Omega_{\chi} h^{2}} .
\end{aligned}
$$

Here $\mu_{r}=m_{N} m_{\chi} /\left(m_{N}+m_{\chi}\right)$ is a reduced mass with $m_{N}$ being the nucleon mass. As the dark matter is much heavier than the nucleon, we can simply read

$\sigma_{\chi N}=2.9 \times 10^{-45} \mathrm{~cm}^{2}\left(\frac{\mu_{r}}{940 \mathrm{MeV}}\right)^{2}\left(\frac{0.11}{\Omega_{\chi} h^{2}}\right)\left(\frac{4 \mathrm{TeV}}{m_{\chi}}\right)^{2}$.

To match the dark matter direct detection results [30,31] into account, the dark matter mass should have a more stringent low limit,

$$
m_{\chi} \gtrsim 4 \mathrm{TeV}
$$

So, the dark matter mass range (44) should be modified by

$$
\begin{aligned}
4 \mathrm{TeV}\left(\frac{0.11}{\Omega_{\chi} h^{2}}\right)^{\frac{1}{2}} \lesssim m_{\chi}<34 \mathrm{TeV}\left(\frac{0.11}{\Omega_{\chi} h^{2}}\right)^{\frac{1}{2}} \\
\text { for } 21 \mathrm{TeV} \lesssim\langle\xi\rangle<61 \mathrm{TeV} .
\end{aligned}
$$

Furthermore, the dark matter annihilation products in Eq. (39) could be also constrained by the dark matter indirect searches such as the gamma-ray observations of dwarf galaxies from the Fermi-LAT satellite. We find our dark matter fermion with the mass (48) and the annihilation cross section $\left\langle\sigma_{\mathrm{A}} v_{\text {rel }}\right\rangle \simeq 0.1 \mathrm{pb}$ can be allowed by the experimental results [32].

We also check if the massless $\zeta_{R}$ fermion can decouple above the QCD scale to satisfy the BBN constraint on the effective neutrino number. For this purpose, we need consider the annihilations of the $\zeta_{R}$ fermion into the relativistic species at the QCD scale,

$$
\begin{aligned}
\sigma_{\zeta} & =\sum_{f=d, u, s, e, \mu, \nu_{L}} \sigma\left(\zeta_{R}+\zeta_{R}^{c} \rightarrow f+f^{c}\right) \\
& =\frac{6 g_{B-L}^{4}}{\pi} \frac{s}{M_{Z_{B-L}}^{4}}=\frac{3}{2 \pi} \frac{s}{\langle\xi\rangle^{4}},
\end{aligned}
$$

with $s$ being the Mandelstam variable. The interaction rate then should be [11]

$$
\Gamma_{\zeta}=\frac{\frac{T}{32 \pi^{4}} \int_{0}^{\infty} s^{3 / 2} K_{1}\left(\frac{\sqrt{s}}{T}\right) \sigma_{\zeta} d s}{\frac{2}{\pi^{2}} T^{3}}=\frac{18}{\pi^{3}} \frac{T^{5}}{\langle\xi\rangle^{4}},
$$

with $K_{1}$ being a Bessel function. By requiring

$$
\Gamma_{\zeta}=H(T)
$$

the decoupling temperature of the dark radiation $\zeta_{R}$ can be given by

$$
T_{\zeta}=\frac{1}{3} \pi^{\frac{3}{2}}\left[\frac{1}{5} g_{*}\left(T_{\zeta}\right)\right]^{\frac{1}{6}}\left(\frac{\langle\xi\rangle}{M_{\mathrm{Pl}}}\right)^{\frac{1}{3}}\langle\xi\rangle .
$$

The contribution of the dark radiation $\zeta_{R}$ to the effective neutrino number then should be [22]

$$
\Delta N_{\nu}=\left[\frac{10.75}{g_{*}\left(T_{\zeta}\right)}\right]^{\frac{4}{3}}
$$

We take $g_{*}(300 \mathrm{MeV}) \simeq 61.75[22]$ and then find

$T_{\zeta} \simeq 300 \mathrm{MeV}$ and $\Delta N_{\nu} \simeq 0.098$ for $\langle\xi\rangle=11 \mathrm{TeV}$.

So, the massless $\zeta_{R}$ fermion should be harmless for the parameter choice (48).

\section{CONCLUSION}

In this paper we have shown a $U(1)_{B-L}$ gauge symmetry can provide the lepton number violation for the Majorana neutrino mass generation, meanwhile, can predict the existence and guarantee the stability of the dark matter. Specifically, because of their Yukawa couplings to the Higgs singlet for spontaneously breaking the $U(1)_{B-L}$ symmetry, three right-handed neutrinos without any Yukawa couplings to the SM can form a Dirac fermion 
besides a massless state. The massive right-handed neutrinos can serve as a stable dark matter particle while the massless one decouples safely. On the other hand, after this $U(1)_{B-L}$ symmetry breaking, two or more heavy Higgs singlets can acquire their small VEVs to induce the cubic coupling between the type II seesaw Higgs triplet and the SM Higgs doublet. Therefore, the left-handed neutrinos can naturally obtain their tiny Majorana masses even if the Higgs triplet is at the $\mathrm{TeV}$ scale. Through the interactions for generating the neutrino masses, the heavy Higgs singlets can decay to produce a lepton asymmetry stored in the Higgs triplet. The sphaleron processes then can partially transfer this lepton asymmetry to a baryon asymmetry.

\section{ACKNOWLEDGMENTS}

This work was supported by the National Natural Science Foundation of China under Grant No. 11675100 and the Recruitment Program for Young Professionals under Grant No. 15Z127060004.
[1] M. Tanabashi et al. (Particle Data Group), Phys. Rev. D 98, 030001 (2018).

[2] P. Minkowski, Phys. Lett. B 67, 421 (1977); T. Yanagida, in Proceedings of the Workshop on Unified Theory and the Baryon Number of the Universe, edited by O. Sawada and A. Sugamoto (KEK, Tsukuba, 1979), p. 95; M. Gell-Mann, P. Ramond, and R. Slansky, in Supergravity, edited by F. van Nieuwenhuizen and D. Freedman (North Holland, Amsterdam, 1979), p. 315; S. L. Glashow, in Quarks and Leptons, edited by M. Lévy et al. (Plenum, New York, 1980), p. 707; R. N. Mohapatra and G. Senjanović, Phys. Rev. Lett. 44, 912 (1980).

[3] M. Magg and C. Wetterich, Phys. Lett. B 94, 61 (1980); J. Schechter and J. W. F. Valle, Phys. Rev. D 22, 2227 (1980); T. P. Cheng and L. F. Li, Phys. Rev. D 22, 2860 (1980); G. Lazarides, Q. Shafi, and C. Wetterich, Nucl. Phys. B181, 287 (1981); R. N. Mohapatra and G. Senjanović, Phys. Rev. D 23, 165 (1981).

[4] M. Fukugita and T. Yanagida, Phys. Lett. B 174, 45 (1986).

[5] P. Langacker, R. D. Peccei, and T. Yanagida, Mod. Phys. Lett. A 01, 541 (1986); M. A. Luty, Phys. Rev. D 45, 455 (1992); R. N. Mohapatra and X. Zhang, Phys. Rev. D 46, 5331 (1992).

[6] M. Flanz, E. A. Paschos, and U. Sarkar, Phys. Lett. B 345, 248 (1995); M. Flanz, E. A. Paschos, U. Sarkar, and J. Weiss, Phys. Lett. B 389, 693 (1996); L. Covi, E. Roulet, and F. Vissani, Phys. Lett. B 384, 169 (1996); A. Pilaftsis, Phys. Rev. D 56, 5431 (1997).

[7] E. Ma and U. Sarkar, Phys. Rev. Lett. 80, 5716 (1998).

[8] R. Barbieri, P. Creminelli, A. Strumia, and N. Tetradis, Nucl. Phys. B575, 61 (2000).

[9] T. Hambye, Nucl. Phys. B633, 171 (2002).

[10] S. Davidson and A. Ibarra, Phys. Lett. B 535, 25 (2002); W. Buchmüller, P. Di Bari, and M. Plümacher, Nucl. Phys. B665, 445 (2003).

[11] G. F. Giudice, A. Notari, M. Raidal, A. Riotto, and A. Strumia, Nucl. Phys. B685, 89 (2004).

[12] T. Hambye and G. Senjanović, Phys. Lett. B 582, 73 (2004); S. Antusch and S. F. King, Phys. Lett. B 597, 199 (2004).
[13] W. Buchmuller, P. Di Bari, and M. Plumacher, Ann. Phys. (Amsterdam) 315, 305 (2005).

[14] V. A. Kuzmin, V. A. Rubakov, and M. E. Shaposhnikov, Phys. Lett. 155B, 36 (1985).

[15] A. Abada, C. Biggio, F. Bonnet, M. B. Gavela, and T. Hambye, J. High Energy Phys. 12 (2007) 061.

[16] P. Fileviez Perez, T. Han, G. Huang, T. Li, and K. Wang, Phys. Rev. D 78, 015018 (2008).

[17] P. H. Gu, H. J. He, U. Sarkar, and X. Zhang, Phys. Rev. D 80, 053004 (2009).

[18] J. C. Montero and V. Pleitez, Phys. Lett. B 675, 64 (2009).

[19] S. Patra, W. Rodejohann, and C. E. Yaguna, J. High Energy Phys. 09 (2016) 076.

[20] S. K. Majee and N. Sahu, Phys. Rev. D 82, 053007 (2010).

[21] J. McDonald, N. Sahu, and U. Sarkar, J. Cosmol. Astropart. Phys. 04 (2008) 037.

[22] E. W. Kolb and M. S. Turner, The Early Universe (AddisonWesley, Reading, MA, 1990).

[23] J. A. Harvey and M. S. Turner, Phys. Rev. D 42, 3344 (1990).

[24] M. Carena, A. Daleo, B. A. Dobrescu, and T. M. P. Tait, Phys. Rev. D 70, 093009 (2004).

[25] S. Alioli, M. Farina, D. Pappadopulo, and J. T. Ruderman, Phys. Rev. Lett. 120, 101801 (2018).

[26] M. Klasen, F. Lyonnet, and F. S. Queiroz, Eur. Phys. J. C 77, 348 (2017).

[27] P. Fileviez Perez, C. Murgui, and A. D. Plascencia, Phys. Rev. D 100, 035041 (2019).

[28] M. Beltran, D. Hooper, E. W. Kolb, and Z. C. Krusberg, Phys. Rev. D 80, 043509 (2009); K. Cheung, P. Y. Tseng, and T. C. Yuan, J. Cosmol. Astropart. Phys. 01 (2011) 004.

[29] G. Jungman, M. Kamionkowski, and K. Griest, Phys. Rep. 267, 195 (1996).

[30] X. Cui et al. (PandaX-II Collaboration), Phys. Rev. Lett. 119, 181302 (2017).

[31] E. Aprile et al. (XENON Collaboration), Phys. Rev. Lett. 121, 111302 (2018).

[32] D. Hooper, Proc. Sci., TASI2018 (2019) 010. 